JOURNAL OF INTEGRAL EQUATIONS

AND APPLICATIONS

Volume 4, Number 2, Spring 1992

\title{
SWITCHING BEHAVIOR OF PN-DIODES: VOLTERRA INTEGRAL EQUATION MODELS
}

\author{
RICHARD K. MILLER AND ANDREAS UNTERREITER
}

1. Introduction. PN-diodes are semiconductive devices with a wide range of applications in modern electrical engineering. The purpose of this paper is to study a mathematical model of the current-time characteristics of such devices. We shall investigate an electrical circuit consisting of a PN-diode, a linear Ohmic resistor and a voltage source. These are connected serially. The analysis is restricted to the case where the diode is abruptly switched from the forward to the backward direction.

The standard model which describes the dynamics of a semiconductive device's charge densities is the drift-diffusion equations of Van Roosbroeck [9]. These drift-diffusion equations are a system of coupled partial differential equations in three space dimensions plus time. (The spatial variable ranges over a smooth bounded domain $\Omega$. It may be approximated by an infinite domain in some cases.) The equations are subject to boundary conditions which reflect the assumptions on the diode's contacts with the electrical circuit as well as Kirchhoff's laws. The initial conditions are provided by the solution of the stationary equations which describe the biasing of the circuit in the forward direction before time zero.

It is useful to reduce the number of space dimensions when dealing with PN-diodes possessing spatial symmetries. Such a case of interest here is the one-dimensional model of a $\mathrm{PN}$-diode with piecewise constant parameter and semi-infinite $\mathrm{N}$ and $\mathrm{P}$ regions.

Under simplifying assumptions (zero space charge approximations, low injection limit), the $n$-dimensional ( $n=1,2$ or 3 ) system of partial differential equations can be reduced to a single diffusion equation in $n$ space dimensions plus time. Two cases are considered here.

\footnotetext{
Received by the editors on July 8,1991 .

The first author supported by the National Science Foundation under Grant Number DMS-8901574.

The second author partially supported by the "Fonds zur Förderung der wissenschaftlichen Forschung," Projekt No. S3210.

Copyright (C)1992 Rocky Mountain Mathematics Consortium
} 
(I) If the spatial domains of the $\mathrm{P}$ and $\mathrm{N}$ regions are both finite, then this diffusion equation can be further reduced to a Volterra integral equation with the total current as the unknown.

(II) If the PN-diode has piecewise constant parameters, the $\mathrm{N}$ and $\mathrm{P}$ regions are semi-infinite and the equation has only one space dimension, then this diffusion equation can be further reduced to a system of coupled Volterra integral equations.

The reductions in these two cases are discussed in detail in Schmeiser et al. [6], Schmeiser and Unterreiter [7] and Unterreiter [8].

In Section 2 below we formulate the single Volterra integral equation as well as the system of two coupled Volterra integral equations which come from cases (I) and (II), respectively. We show that in the case of symmetric diodes the system of two equations can be easily decoupled to obtain a single Volterra integral equation which has the same form as the equation obtained in case (I) above. The form of this equation is

$$
\exp (-U-w I)=1+C I_{F}+\left(I-I_{F}\right) * a
$$

where $*$ denotes convolution, e.g.,

$$
I * a(t)=\int_{0}^{t} I(t-s) a(s) d s,
$$

$I(t)$ is the unknown current, $a(t)$ is a completely monotone kernel, $U, w, I_{F}$ and $C$ are positive physical constants and $C=\lim (1 * a)(t)$, $t \rightarrow \infty$.

In Section 3 we present a rigorous theory for the single integral equation case. Our results include existence, uniqueness, global boundedness, monotonicity, and asymptotic behavior of the solution. These results generalize the results of Schmeiser et al. [6] who studied the symmetric, one-dimensional diode with semi-infinite $\mathrm{P}$ and $\mathrm{N}$ regions.

In Section 4 we show that the nonsymmetric form of the problem can be reduced from a coupled system of Volterra equations to a single Volterra equation to which the results of Section 3 will also apply. Hence, we show that the results in $[\mathbf{7}]$ and $[\mathbf{8}]$ can be extended from the symmetric to the nonsymmetric case.

2. The integral equation model. In $[7]$ and $[8]$ it was shown that under the zero space charge approximation and low injection limit, one 
can replace the (degenerate) $n$-dimensional drift diffusion equations by a single Volterra integral equation of the form

$$
\exp (-U-w I)=1+C I_{F}+\left(I-I_{F}\right) * a
$$

Here $I_{F}$ is the constant uniquely determined via the relation

$$
\exp \left(W-w I_{F}\right)=1+C I_{F}
$$

The constants $W$ and $-U$ are the potential drops generated by the voltage source for $t<0$ and, respectively, $t>0$; $w$ denotes the resistance in Ohms in the circuit and $a(t)$ is a completely monotone kernel of the form

$$
a(t)=\sum_{n=1}^{\infty} K_{n}^{2} e^{\lambda_{n} t}
$$

with all $\lambda_{n}$ negative and $\lambda_{n} \rightarrow-\infty$ as $n \rightarrow \infty$. Furthermore, the kernel $a(t)$ is integrable, indeed

$$
C=\sum_{n=1}^{\infty} K_{n}^{2} /\left|\lambda_{n}\right|=\int_{0}^{\infty} a(t) d t<\infty
$$

In the case of a model with one space dimensional and with semiinfinite $\mathrm{N}$ and $\mathrm{P}$ regions, it was shown in $[\mathbf{6}]$ that the currents in the circuit satisfy

$$
\left\{\begin{aligned}
\exp (-U-w I) & =1+C_{N} Z_{N}^{I}+\left(z_{N}-Z_{N}^{I}\right) * a_{N} \\
\exp (-U-w I) & =1+C_{p} Z_{p}^{I}+\left(z_{p}-Z_{p}^{I}\right) * a_{p} \\
I & =z_{N}+z_{p} .
\end{aligned}\right.
$$

The three functions $I, z_{N}$ and $z_{p}$ are unknown currents, $Z_{N}^{I}$ and $Z_{p}^{I}$ are constants uniquely determined by the relations

$$
\begin{aligned}
\exp \left(W-w I_{F}\right) & =1+C_{N} Z_{N}^{I} \\
\exp \left(W-w I_{F}\right) & =1+C_{p} Z_{p}^{I} \\
Z_{N}^{I}+Z_{p}^{I} & =I_{F}
\end{aligned}
$$


and $a_{N}(t)$ and $a_{p}(t)$ are given by

$$
\begin{aligned}
a_{N}(t) & =\frac{C_{N}}{\sqrt{\tau_{p}}} \frac{\exp \left(-t / \tau_{p}\right)}{\sqrt{\pi t}}, \\
a_{p}(t) & =\frac{C_{p}}{\sqrt{\tau_{N}}} \frac{\exp \left(-t / \tau_{N}\right)}{\sqrt{\pi t}}
\end{aligned}
$$

The physical constants $C_{N}, C_{p}, \tau_{N}$ and $\tau_{p}$ are all positive. It is easy to check that

$$
\lim _{t \rightarrow \infty}\left(1 * a_{N}\right)(t)=C_{N} \text { and } \lim _{t \rightarrow \infty}\left(1 * a_{p}\right)(t)=C_{p}
$$

We will call a diode "symmetric" if $\tau_{n}=\tau_{p}$. In the symmetric case there is no loss of generality in taking $\tau_{N}=\tau_{p}=1$. In this case some easy algebraic manipulations can be used to see that (2.2) reduces to

$$
\exp (-U-w I)=1+C I_{F}+\left(I-I_{F}\right) * a
$$

where $a(t)=C e^{-t} / \sqrt{\pi t}, C$ is the positive constant defined via the relation

$$
C=\frac{1}{\frac{1}{C_{N}}+\frac{1}{C_{p}}}
$$

and $I_{F}$ is the unique solution of

$$
\exp \left(W-w I_{F}\right)=1+C I_{F}
$$

The $n$-dimensional finite region model (2.1) and the symmetric model (2.3) both have the form

$$
\exp (-U-w I)=1+C I_{F}+\left(I-I_{F}\right) * a
$$

and

$$
\exp \left(W-w I_{F}\right)=1+C I_{F}
$$

where $a(t)$ is a completely monotone and integrable function such that

$$
\lim _{t \rightarrow \infty}(1 * a)(t)=C>0 .
$$


Solutions of this general model equation will be studied in detail in Section 3. In that analysis we will show that $I(t)$ tends to a limit $I_{\infty}$ as $t \rightarrow \infty$. The constant $I_{\infty}$ is the unique solution of

$$
\exp \left(-U-w I_{\infty}\right)=1+C I_{\infty}
$$

Define $y$ via the relation $y=\exp \left(-w\left(I-I_{\infty}\right)\right)-1$. Then $I(t)-I_{\infty} \rightarrow 0$ as $t \rightarrow \infty$ if and only if $y(t) \rightarrow 0$ as $t \rightarrow \infty$. In terms of $y(t)$ equation (2.4) transforms to a new equation of the form

$$
y=A_{1}(C-1 * a)-B_{1} \ln (y+1) * a
$$

where

$$
A_{1}=\frac{I_{F}-I_{\infty}}{1+C I_{\infty}} \quad \text { and } \quad B_{1}=\frac{1}{w\left(1+C I_{\infty}\right)} .
$$

From (2.5) we see that $1+C I_{\infty}>0$ and that $I_{\infty}$ must be negative. Hence, $B_{1}>0$. From $(2.4 \mathrm{~b})$ and $(2.5)$ we see that $C\left(I_{F}-I_{\infty}\right)=$ $e^{W-w I_{F}}-e^{-U-w I_{\infty}}$. If $I_{F}-I_{\infty} \leq 0$, then $W-w I_{F}>-U-w I_{F} \geq$ $-U-w I_{\infty}$ and $0 \geq C\left(I_{F}-I_{\infty}\right) \geq e^{W-w I_{F}}-e^{-U-w I_{\infty}}>0$, a contradiction. Hence, $I_{F}-I_{\infty}>0$ and the constants $A_{1}$ and $B_{1}$ are both positive.

3. General results. The integral equation (2.6) can be written in the form

$$
y(t)=f(t)-\int_{0}^{t} a(t-s) \ln [y(s)+1] d s,
$$

or in convolution notation $y=f-a * \ln [y+1]$. We will study (3.1) under the following assumptions:

(A1) $a(t) \in L_{\mathrm{loc}}^{1}(0, \infty), a(t) \not \equiv 0$ and $a(t)$ is completely monotone.

Let $r(t)$ denote the resolvent of $a(t)$. Thus, $r(t)$ is the unique locally integrable solution of

$$
r(t)=a(t)-\int_{0}^{t} a(t-s) r(s) d s .
$$

Note that $r(t) \not \equiv 0$ and $r(t) \in L_{\mathrm{loc}}^{1}(0, \infty)$. 
(A2) $f(t)$ is a nonnegative function in $L_{\mathrm{loc}}^{1}(0, \infty)$

(A3) $z=(\delta-r) * f$ is nonnegative, i.e., the unique solution of

$$
z(t)=f(t)-\int_{0}^{t} a(t-s) z(s) d s
$$

is nonnegative.

(A4) $f \in C[0, \infty)$ and the $\operatorname{limit}_{\lim _{t \rightarrow \infty}} f(t)=F$ exists and is nonnegative.

(A5) $f \in C^{1}(0, \infty), f^{\prime} \in L^{1}(0,1)$ and $(\delta-r) * f^{\prime} \leq 0$.

General information on completely monotone functions can be found in Widder [10, Chapter 4]. Information on the resolvents of completely monotone functions can be found in Miller [4, Chapter IV, Section 7] or G. Gripenberg, S.O. Londen and D. Staffans, [2, Chapter 5], see also Gripenberg [1]. In particular, as Reuter [5] has shown, the resolvent $r(t)$ will also be completely monotone. Moreover, if $a \in L^{1}(0, \infty)$, then

$$
\int_{0}^{\infty} r(t) d t=\frac{\int_{0}^{\infty} a(s) d s}{1+\int_{0}^{\infty} a(s) d s}<1
$$

If $a \notin L^{1}(0, \infty)$, then $\int_{0}^{\infty} r(t) d t=1$. Local existence, uniqueness and continuation results for (3.1) or (3.2) can also be found in [2] or [4]. Such results will be used as needed without further comment.

Theorem 1. If (A1-A3) are true, then the solution $y(t)$ of (3.1) exists for all $t \geq 0$ and $0 \leq y(t) \leq f(t)$ for all $t \geq 0$.

Proof. The local solution of (3.1) can be continued so long as it remains bounded. We will show this boundedness. Let $\delta(t)$ be the delta function. Convolution multiply $(3.1)$ by $(\delta-r)$ where $r(t)$ is the resolvent of $a(t)$. Then

$$
y=(\delta-r) * f+r *(y-\ln [y+1])
$$

or

$$
y=z+r *(y-\ln [y+1])
$$


for as long as $y(t)$ exists. Since $\ln (w)$ is a strictly concave function of $w$, then $y-\ln [y+1]>0$ for all $y \neq 0$. Also, $z(t) \geq 0$ by (A3) and $r(t)>0$ since it is completely monotone. Thus the solution of (3.3) is nonnegative for as long as it exists. But $y(t) \geq 0$ implies $\ln [y(t)+1] \geq 0$ for as long as $y(t)$ exists. Use this and (A2) in (3.1) to see that $y(t) \leq f(t)$ for as long as $y(t)$ exists. By continuation, $y(t)$ will exist for all $t \geq 0$ and $0 \leq y(t) \leq f(t)$ for all $t \geq 0$. This proves Theorem 1.

Theorem 2. If (A1-A4) are true, then the limit $\lim _{t \rightarrow \infty} y(t)=y^{*}$ exists. If $a(t) \notin L^{1}(0, \infty)$, then $y^{*}=0$. Otherwise, $y^{*}$ is the unique solution of

$$
y^{*}=F-\left(\int_{0}^{\infty} a(s) d s\right) \ln \left[y^{*}+1\right]
$$

Proof. Under our hypotheses we see from Theorem 1 that $0 \leq y(t) \leq$ $f(t)$ for all $t \geq 0$. Hence, $y(t)$ is bounded and results on limiting equations apply, cf., e.g., [4, Chapter III, Theorem 7.1]. The positive limit set of $y(t)$ is an invariant set with respect to the equation

$$
y_{1}(t)=z_{\infty}+\int_{-\infty}^{t} r(t-s)\left(y_{1}(s)-\ln [y(s)+1]\right) d s,
$$

where $z_{\infty}=\operatorname{limit} z(t)$ as $t \rightarrow \infty$. If $a \notin L^{1}(0, \infty)$, then $\int_{0}^{\infty} r(s) d s=1$ and

$$
z_{\infty}=\lim _{t \rightarrow \infty}\left(f(t)-\int_{0}^{t} r(t-s) f(s) d s\right)=F-\int_{0}^{\infty} r(s) F d s=0 .
$$

If $a \in L^{1}(0, \infty)$, then

$$
z_{\infty}=F\left(1-\int_{0}^{\infty} r(s) d s\right) \geq 0 .
$$

Let $y^{*}$ solve (3.4) so that $y^{*}=0$ if $z_{\infty}=0$ and $y^{*}>0$ if $z_{\infty}>0$. Then

$$
y^{*}=z_{\infty}+\int_{-\infty}^{t} r(t-s)\left(y^{*}-\ln \left[y^{*}+1\right]\right) d s
$$


and $y_{2}=y_{1}-y^{*}$ will solve

$$
\begin{aligned}
y_{2}(t) & =\int_{-\infty}^{t} r(t-s)\left(y_{1}(s)-\ln \left[y_{1}(s)+1\right]-y^{*}+\ln \left[y^{*}+1\right]\right) d s \\
& =\int_{-\infty}^{t} r(t-s)\left(y_{2}(s)-\ln \left[\left(y_{2}(s)+y^{*}+1\right) /\left(y^{*}+1\right)\right]\right) d s
\end{aligned}
$$

or

$$
y_{2}(t)=\int_{-\infty}^{t} r(t-s) H\left(y_{2}(s)\right) d s
$$

where $H(y)=y-\ln \left[\left(y+y^{*}+1\right) /\left(y^{*}+1\right)\right]$. Note that $H(0)=0$ and $0 \leq H^{\prime}(y) \leq 1$ when $y+y^{*} \geq 0$.

Consider the case $a \in L^{1}(0, \infty)$. Then

$$
R=\int_{0}^{\infty} r(s) d s<1 .
$$

Since the solution $y(t)$ is nonnegative, then all points in its positive limit set are nonnegative. Hence, there is no loss of generality in assuming that the solution $y_{1}(t)$ of (3.5) is nonnegative. Thus, $y_{2}(t)+y^{*}=$ $y_{1}(t) \geq 0$ in our case and $0 \leq H^{\prime}\left(y_{2}(s)\right) \leq 1$ for all $s$. This means that for any $t$ in $(-\infty, \infty)$, one has

$$
\begin{aligned}
\left|y_{2}(t)\right| & \leq \int_{-\infty}^{t} r(t-s)\left|H_{2}\left(y_{2}(s)\right)\right| d s \\
& \leq \int_{-\infty}^{t} r(t-s)\left|y_{2}(s)\right| d s \\
& \leq R \sup _{s}\left|y_{2}(s)\right| d s .
\end{aligned}
$$

Hence,

$$
\sup _{t}\left|y_{2}(t)\right| \leq R \sup _{s}\left|y_{2}(s)\right| .
$$

Since $R<1$, this is only possible if $y_{2}(t) \equiv 0$. Hence, $y_{1}(t) \equiv y^{*}$ is the only nonnegative and bounded solution of (3.5) and the positive limit set of $y(t)$ must consist of the single point $y^{*}$. Since $y(t)$ must tend to its positive set, then $a \in L^{1}(0, \infty)$ implies $\lim _{t \rightarrow \infty} y(t)=y^{*}$. 
Next, consider the case where $a \notin L^{1}(0, \infty)$. In this case, $y^{*}=0$. Assume that the nonnegative, bounded solution $y(t)$ of $(3.1)$ does not tend to zero as $t \rightarrow \infty$. There must be a sequence $t_{n} \rightarrow \infty$ and a $\bar{y}>0$ such that

$$
\lim _{n \rightarrow \infty} y\left(t_{n}\right)=\bar{y}=\lim _{t \rightarrow \infty} \sup y(t) .
$$

By Theorem 2.1 in [4, Chapter III] there is a subsequence, which we may assume is the entire sequence, and a solution of (3.5) such that

$$
\lim _{n \rightarrow \infty} y\left(t+t_{n}\right)=y_{1}(t), \quad-\infty<t<\infty
$$

This solution of (3.5) will satisfy the relation $y_{1}(0)=\bar{y}=\sup \left\{y_{1}(s)\right.$ : $-\infty<s<\infty\}$. Since $y_{1}(t)$ is continuous, then $y_{1}(t)>0$ in a neighborhood of $t=0$. Thus, $H\left(y_{2}(s)\right)<1$ on a set $0<s<\delta$ for some $\delta>0$. Since $r(t)$ is completely monotone, then it is analytic for Ret $>0$. Since also $r(t) \not \equiv 0$ for $t>0$, then $r(t)>0$ for $t>0$. By (3.6), we see that

$$
\begin{aligned}
\bar{y}=y_{1}(0) & =\int_{-\infty}^{0} r(-s) H\left(y_{1}(s)\right) d s \\
& <\int_{-\infty}^{0} r(-s)\left|y_{1}(s)\right| d s \\
& \leq \int_{-\infty}^{0} r(-s) \bar{y} d s=\bar{y},
\end{aligned}
$$

a contradiction. Hence, $y(t) \rightarrow 0$ as $t \rightarrow \infty$. This proves Theorem 2 . $\square$

Theorem 3. If (A1-A5) are true, then $y(t)$ is a nonincreasing function of $t$.

Proof. By Theorems 1 and 2 above, the solution $y(t)$ of (3.1) is nonnegative and tends to $y^{*}$ as $t \rightarrow \infty$. By [2, Theorem 13.3.3] $y(t)$ is absolutely continuous with derivative $w(t)$ satisfying the equation

$$
w(t)=f^{\prime}(t)-a(t) \ln [f(0)+1]-\int_{0}^{t} a(t-s)[w(s) /(1+y(s))] d x
$$


We will show that $w(t) \leq 0$ so that $y(t)$ is nonincreasing in $t$. Convolution multiply $(3.7)$ by $(\delta-r)$ to obtain $w=(\delta-r) * f^{\prime}-\ln [f(0)+1] r+$ $r *(1-1 /(1+y)) w$. Since $(\delta-r) * f^{\prime} \leq 0$ by $(\mathrm{A} 5), \ln [f(0)+1]>0$, $r(t)>0$ and $1-(1+y)^{-1}>0$, then $w$ is nonpositive. This proves Theorem 3 .

All of the results in this section can be applied to equation (2.6). Indeed, $f(t)=A_{1}(C-1 * a)$ is positive and decreasing to zero as $t \rightarrow \infty$. If $r(t)$ is the resolvent of $a(t)$, then $\int_{0}^{\infty} a(s) d s=C$ and $\int_{0}^{\infty} r(s) d s=C(1+C)^{-1}$. Hence,

$$
\begin{aligned}
z & =(\delta-r) * f=A_{1}[(\delta-r) * C-(\delta-r) * a * 1] \\
& =A_{1}\left[C-C \int_{0}^{t} r(s) d s-r * 1\right] \\
& =A_{1}\left[C-(C+1) \int_{0}^{t} r(s) d s\right] \geq 0 .
\end{aligned}
$$

Assumptions (A1-A5) are all true for (2.6).

Remark. Equation (3.1) has the form $y=f-a * h(y)$ where $a(t)$ is completely monotone and $h(y)$ is a $C^{1}$, increasing function with $h(0)=0$. Such nonlinear equations have been studied in several papers, cf., e.g., [1, 2 or 4]. The key ingredient which makes our results new and different is that $h(y)=\ln [y+1]$ is also a concave function. All of our results remain true if for any nonlinear function $h(y)$ which is $C^{1}$, increasing, concave and satisfies $h(0)=0$.

4. The dual integral equations. The coupled system (2.2) formulated in Section 2 can be written in the form

$$
\left\{\begin{aligned}
e^{-U-w I} & =1+C_{N} Z_{N}^{I}+\left(z_{N}-Z_{N}^{I}\right) * a_{N} \\
e^{-U-w I} & =1+C_{p} Z_{p}^{I}+\left(z_{p}-Z_{p}^{I}\right) * a_{p} \\
I & =z_{N}+z_{p}
\end{aligned}\right.
$$

where

$$
a_{N}(t)=C_{N} \sqrt{\gamma_{n}} \frac{e^{-\gamma_{n} t}}{\sqrt{\pi t}}, \quad a_{p}(t)=C_{p} \sqrt{\gamma_{p}} \frac{e^{-\gamma_{p} t}}{\sqrt{\pi t}}
$$


and $U, w, C_{N}, C_{p}, \gamma_{N}$ and $\gamma_{p}$ are positive constants. The constants $Z_{N}^{I}, Z_{p}^{I}$ and $I_{F}$ satisfy the steady state problem

$$
\left\{\begin{array}{rl}
e^{W-w I_{F}} & =1+C_{N} Z_{N}^{I} \\
e^{W-w I_{F}} & =1+C_{p} Z_{p}^{I} \\
I_{F} & =Z_{p}^{I}+Z_{N}^{I}
\end{array} .\right.
$$

Solve the first two equations for $Z_{N}^{I}$ and $Z_{p}^{I}$. Then

$$
I_{F}=\left(e^{W-w I_{F}}-1\right) C_{N}^{-1}+\left(e^{W-w I_{F}}-1\right) C_{p}^{-1}
$$

or

$$
I_{F}=\left(e^{W-w I_{F}}-1\right) \frac{1}{C}, \quad \frac{1}{C}=\frac{1}{C_{N}}+\frac{1}{C_{p}} .
$$

Thus,

$$
e^{W-w I_{F}}=1+C I_{F}, \quad C=\frac{C_{N} C_{p}}{C_{N}+C_{p}} .
$$

Similarly, let $I_{\infty}, Z_{p}^{\infty}$ and $Z_{N}^{\infty}$ solve

$$
\begin{aligned}
e^{-U-w I_{\infty}} & =1+C_{N} Z_{N}^{\infty}=1+C_{p} Z_{p}^{\infty} \\
I_{\infty} & =Z_{N}^{\infty}+Z_{p}^{\infty}
\end{aligned}
$$

so that

$$
e^{-U-w I_{\infty}}=1+C I_{\infty}
$$

Notice that $1+C I_{F}>0,1+C I_{\infty}>0$ and $I_{F}-I_{\infty}>0$.

Let $z_{N_{1}}=z_{N}-Z_{n}^{I}$ and $z_{p 1}=z_{p}-Z_{p}^{I}$. Let $\hat{Z}_{N 1}$ and $\hat{Z}_{p 1}$ denote their Laplace transforms. From the first two equations in (4.1) we see that

$$
\hat{Z}_{N 1} \frac{A_{N}}{\sqrt{s+\gamma_{N}}}=\hat{Z}_{p 1} \frac{A_{p}}{\sqrt{s+\gamma_{p}}},
$$

where $\sqrt{ }$ means the principal root cut on the negative real axis while $A_{N}=C_{N} \sqrt{\gamma_{n}}$ and $A_{p}=C_{p} \sqrt{\gamma_{p}}$. Thus,

$$
\hat{Z}_{N 1}=\frac{A_{p}}{A_{N}} \frac{\sqrt{s+\gamma_{N}}}{\sqrt{s+\gamma_{p}}} \hat{Z}_{p 1}=\frac{A_{p}}{A_{N}}\left(1+\hat{\beta}_{0}(s)\right) \hat{Z}_{p 1}
$$


where $\beta_{0}(t)$ is that locally integrable function such that

$$
\hat{\beta}_{0}(s)=\frac{\sqrt{s+\gamma_{N}}}{\sqrt{s+\gamma_{p}}}-1=\frac{\gamma_{N}-\gamma_{p}}{\sqrt{s+\gamma_{p}}} \frac{1}{\sqrt{s+\gamma_{p}}+\sqrt{s+\gamma_{N}}} .
$$

Hence,

$$
\begin{aligned}
I-I_{F} & =\left(z_{N}+z_{p}\right)-\left(Z_{N}^{I}+Z_{p}^{I}\right)=z_{N 1}+z_{p 1} \\
& =\frac{A_{p}}{A_{N}}\left(\delta+\beta_{0}\right) * z_{p 1}+z_{p 1}
\end{aligned}
$$

or

$$
I-I_{F}=\frac{A_{N}+A_{p}}{A_{N}}\left(\delta+\frac{A_{p}}{A_{N}+A_{p}} \beta_{0}\right) * z_{p 1} .
$$

We use (4.4) in the middle equation of (4.1) to see that

$e^{-U-w I}=1+C_{p} Z_{p}^{I}+a_{p} *\left(\frac{A_{N}}{A_{N}+A_{p}}\right)\left(\delta+\frac{A_{p}}{A_{N}+A_{p}} \beta_{0}\right)^{-1} *\left(I-I_{F}\right)$.

The left side of (4.5) can be written as

$$
\begin{aligned}
e^{-U-w I} & =e^{-U-w I_{\infty}} e^{-w\left(I-I_{\infty}\right)} \\
& =\left(1+C I_{\infty}\right) e^{-w\left(I-I_{\infty}\right)} .
\end{aligned}
$$

Note also that $1+C_{p} Z_{p}^{I}=e^{W-w I_{F}}=1+C I_{F}$. Hence, (4.5) can be rewritten as

$$
\begin{aligned}
e^{-w\left(I-I_{\infty}\right)}-1 & =\frac{1+C I_{F}}{1+C I_{\infty}}-1+\frac{1}{1+C I_{\infty}} \beta_{1} *\left(I-I_{F}\right) \\
& =\frac{1}{1+C I_{\infty}}\left[C\left(I_{F}-I_{\infty}\right)+\beta_{1} *\left(I-I_{F}\right)\right] \\
& =\frac{1}{1+C I_{\infty}}\left[C\left(I_{F}-I_{\infty}\right)+\beta_{1}^{*}\left(I-I_{\infty}+I_{\infty}-I_{F}\right)\right]
\end{aligned}
$$

where

$$
\beta_{1}=\frac{A_{N}}{A_{N}+A_{p}} a_{p} *\left(\delta+\frac{A_{p}}{A_{N}+A_{p}} \beta_{0}\right)^{-1} .
$$


Hence,

(4.6) $e^{-w\left(I-I_{\infty}\right)}-1=\frac{I_{F}-I_{\infty}}{1+C I_{\infty}}\left(C-\beta_{1} * 1\right)+\frac{1}{1+C I_{\infty}} \beta_{1} *\left(I-I_{\infty}\right)$.

Notice that

$$
\hat{\beta}_{1}(s)=\frac{A_{N}}{A_{N}+A_{p}} \frac{A_{p}}{\sqrt{s+\gamma p}}\left(1+\frac{A_{p}}{A_{N}+A_{p}}\left[\frac{\sqrt{s+\gamma N}}{\sqrt{s+\gamma_{p}}}-1\right]\right)^{-1}
$$

or

$$
\hat{\beta}_{1}(s)=\frac{A_{N} A_{p}}{A_{N} \sqrt{s+\gamma_{p}}+A_{p} \sqrt{s+\gamma N}} .
$$

Since $A_{N}=C_{n} \sqrt{\gamma N}$ and $A_{p}=C_{p} \sqrt{\gamma_{p}}$, if $\beta_{1} \in L^{1}(0, \infty)$, then

$$
\int_{0}^{\infty} \beta_{1}(s) d s=\hat{\beta}_{1}(0)=\frac{C_{N} C_{p}}{C_{N}+C_{p}}=C .
$$

This proves the following result.

Theorem 4. System (4.1)-(4.2) reduces, under the transformation $y(t)=e^{-w\left(I(t)-I_{\infty}\right)}-1$, to one Volterra integral equation

$$
y(t)=A_{1}\left(C-\int_{0}^{t} \beta_{1}(s) d s\right)-A_{2} \int_{0}^{t} \beta_{1}(t-s) \ln [y(s)+1] d s
$$

where $A_{1}>0, A_{2}>0$ and $\beta_{1}(t)$ is a locally $L^{1}$ function which satisfies (4.7).

The function $\beta_{1}(t)$ turns out to be completely monotone and integrable over $(0, \infty)$. Indeed, the following result follows from $[\mathbf{2}$, Theorem 5.2.6].

Lemma 5. If $A, B, a$ and $b$ are all positive constants and if $\beta(t)$ is that function whose Laplace transform is

$$
\hat{\beta}(s)=\frac{1}{A \sqrt{s+a}+B \sqrt{s+b}},
$$


then $\beta(t)$ is completely monotone and $L^{1}(0, \infty)$.

Notice that when $a=b>0$, then Lemma 5 is true but trivial. In this case

$$
\hat{\beta}(s)=\frac{1}{(A+B) \sqrt{s+a}}
$$

so that

$$
\beta(t)=\frac{1}{A+B} \frac{e^{-a t}}{\sqrt{\pi t}} .
$$

This function is obviously completely monotone and integrable over $(0, \infty)$. The case $a=b$ corresponds to the symmetric case studied in $[6]$ and $[8]$.

Since $\beta_{1}(t)>0, \beta_{1} \in L^{1}(0, \infty)$ and (4.8) is true, then the following Theorem is clear.

Theorem 6. In (4.9), the function

$$
f(t)=A_{1}\left(C-\int_{0}^{t} \beta_{1}(s) d s\right)
$$

is positive, continuous and decreasing with $f(t) \rightarrow 0$ as $t \rightarrow \infty$.

The next result completes the task of showing that (4.9) satisfies all hypotheses (A1)-(A5) in Section 2.

Theorem 7. In (4.9), let $f$ be defined by (4.10), let a $(t)=A_{2} \beta_{1}(t)$ and let $r(t)$ be the resolvent of $a(t)$. Then the solution $z$ of the linear problem

$$
z(t)=f(t)-\int_{0}^{t} a(t-s) z(s) d s
$$

is nonnegative.

Proof. Here $\beta_{1}(t)=a(t) / A_{2}$. Since $r=a-a * r$, then

$$
z=(\delta-r) * f=A_{1}(\delta-r) *\left(C-\beta_{1} * 1\right) .
$$


Without loss of generality, take $A_{1}=1$. Then

$$
\begin{aligned}
z(t) & =C-C * r-(\delta-r) *\left(A_{2} \beta_{1}\right) * A_{2}^{-1} \\
& =C-C * r-r * A_{2}^{-1} \\
& =C-\left(C+A_{2}^{-1}\right) \int_{0}^{t} r(s) d s .
\end{aligned}
$$

Now $z(0)=C>0$ and $z^{\prime}(t)=-\left(C+A_{2}^{-1}\right) r(t)<0$ for $t>0$ so that the limit $z(\infty)$ will exist. But $r \in L^{1}(0, \infty)$ and

$$
\int_{0}^{\infty} r(s) d s=\frac{\int_{0}^{\infty} A_{2} \beta_{1}(s) d s}{1+\int_{0}^{\infty} A_{2} \beta_{1}(s) d s}=\frac{A_{2} C}{1+A_{2} C} .
$$

Thus,

$$
z(\infty)=C-\frac{A_{2} C+1}{A_{2}} \int_{0}^{\infty} r(s) d s=C-C=0 .
$$

It follows that $z(t)>0$ for all $t \geq 0$. This proves Theorem 7 .

Since all of the necessary hypotheses are true, then the results in Section 3 apply to (4.9). We see that $y(t)$ exists for all $t \geq 0$, is positive and decreases to zero as $t \rightarrow \infty$. Hence, $I(t)$ in (4.5) (or in (4.1)) exists for all $t \geq 0$, is negative and increases to the limit $I_{\infty}$ as $t \rightarrow \infty$.

\section{REFERENCES}

1. Gustaf Gripenberg, On positive, nonincreasing resolvents of Volterra equations, J. Differential Equations 30 (1978), 380-390.

2. G. Gripenberg, S.O. Londen and D. Staffans, Volterra integral and function equations, Cambridge University Press, Cambridge, MA (1990).

3. R.K. Miller, On Volterra integral equations with nonnegative integrable resolvents, J. Math. Anal. Appl. 22 (1968), 319-340.

4. - Nonlinear Volterra integral equations, W.A. Benjamin, Menlo Park, California, 1971.

5. G.E.H. Reuter, Über eine Volterrasche Integralgleichung mit Total Monotonem Kern, Arch. Math. 7 (1958), 59-66.

6. Ch. Schmeiser, A. Unterreiter and R. Weiss, The switching behavior of a PNdiode in the case of low injection, to appear.

7. Ch. Schmeiser and A. Unterreiter, A transient three-dimensional analysis of bipolar semiconductive devices in the case of low injection, to appear. 
8. A. Unterreiter, The Switching behavior of a PN-diode in the case of low injection, Thesis, TU Vienna, 1990.

9. W.V. Van Roosbroeck, Theory of flow of electrons and holes in germanium and other semiconductors, Bell System Tech. J. 29 (1950), 560-607.

10. D.V. Widder, The Laplace transform, Princeton University Press, Princeton, NJ, 1946.

Mathematics Department, Iowa State University, Iowa 50011

Fachbereich 3, Technical University of Berlin, Berlin, Federal RepubLiC OF Germany 1000 